\title{
Emaciation as a Manifestation of Acanthocephaliasis in the Common Eider (Somateria mollissima)
}

\author{
Nadia Stegeman, DVM ${ }^{1}$, Emily Christiansen, DVM ${ }^{2}$, Mark Pokras, DVM ${ }^{3}$ \\ ${ }^{1}$ Texas A\&M University, College Station, Texas \\ 2 Ocean State Veterinary Specialists, East Greenwich, Rhode Island \\ ${ }^{3}$ Tufts Cummings School of Veterinary Medicine, North Grafton, Massachusetts
}

Keywords: acanthocephalan, common eider, mortality events, emaciation

\section{INTRODUCTION}

An annual die-off of juvenile common eiders (Somateria mollissima) occurs during late winter/early spring in coastal New England. Abnormally high mortality events, including significant numbers of adult birds, have occurred concurrently with die-offs of eiders' primary food source, blue mussels (Mytilus edulis). In these situations, eiders are forced to switch to less preferred prey such as shore crabs (Carcinus and Hemigrapsus spp.). This species of shore crabs are a known intermediate host for acanthocephalan parasites (thorny-headed worms). Severe emaciation following infection with the acanthocephalan (Polymorphus botulus) is the common finding of an elevated mortality event in spring of 2006. This paper describes clinical presentations of eiders found during annual die-off periods and discusses possible treatment approaches identified in literature.

Nadia Stegeman is a graduate of Tufts DVM/MPH combined degree program and worked with the Seabird Ecological Assessment Network (SEANET) to investigate causes of mass mortalities on Cape Cod. She is currently a zoo medicine/surgery intern at Texas A\&M University.

Emily Christiansen is a graduate of Tufts DVM/MPH combined degree program and worked with the Seabird Ecological Assessment Network (SEANET) to investigate causes of mass mortalities on Cape Cod. She is currently a small animal rotating intern at Ocean State Hospital in Rhode Island.

Mark Pokras is the director of the wildlife clinic at Tufts University. He is heavily involved in conservation medicine and continues to inspire veterinary students to follow their hearts and strive to better our world.

\section{BACKGROUND}

Polymorphus botulus (P. botulus) infection that leads to severe emaciation and death, is documented as a recurring cause of death in juvenile birds in European wintering ranges of common eider (Camphuysen et al 2002). This parasite commonly infects marine birds, especially diving ducks, as natural intermediate hosts, small crabs and other aquatic invertebrates, are common prey species. Once the larvae are ingested via the intermediate host, the thorny-headed worm matures and the adult worm everts its head, ringed with sharp hooks, to embed itself into the wall of the bird's small intestine. There, the worms remain attached and absorb protein and nutrients passing through the intestine, contributing to malnutrition and emaciation in infected birds. Acanthocephalans do not ingest blood of the host, but blood loss may result from damage to intestinal walls. In some cases worms burrow completely through the intestinal wall, causing perforation and peritonitis (inflammation of the membrane lining the coelom). Eiders normally remain infected with acanthocephalans throughout the remainder of the adult worm's life, although natural starvation has been seen to reduce numbers of acanthocephalans in nesting females with good body condition (Thompson 1985). It is thought that while the parasites are deprived of nutrition during starvation as little is introduced into the digestive system, the female ducks can live off their body's fat reserves.

A low level of infection with P. botulus is an incidental finding on necropsy of common eiders, with abundance of worms varying by age of the bird, season, and year. Infection rate tends to peak in late fall and abundance of worms may differ up to sevenfold between years. Densities of the parasite may be as much as 10 times greater in juveniles compared to 
adult eiders. There is currently no scientific evidence to explain why this occurs; however it is thought that dietary selections may be modified as the bird matures. The numerical burden of worms is not predictive of prognosis, as birds in good body condition may maintain hundreds of acanthocephalans within their small intestine without presenting clinical signs of infection (Thompson 1985). In most years, incidence of death resulting from heavy acanthocephalan infestation is thought to be relatively inconsequential to US and European eider populations.

Improbably, light infections of acanthocephalans may be beneficial to the host as the worms tend to concentrate heavy metals, including lead, at much higher levels than those found in the host's tissues (Sures et al 2002). However, any level of infection may be suboptimal if acanthocephalans compete with the bird for essential elements, as seen in infected fish and mammals (Sures 2000). It is speculated that as emaciation develops, muscle and fat breakdown releases stored lipophilic (fat-soluble) toxins that may be found at higher levels in the circulation of these emaciated birds (Kuiken 2001). Clearly, any factor compromising the immune system and strength of an individual may decrease tolerance of parasite burden and predispose the bird to developing clinical signs with a lighter infection than an uncompromised animal.

\section{MASS MORTALITIES OF COMMON EIDERS}

Mass mortalities of eiders were reported in several populations corresponding to a die-off of the blue mussel. In Europe, mussel populations have declined when over-harvested or in times of suboptimal water temperatures and/or nutrient levels (Camphuysen et al 2002). During a recent eider die-off (2006) in Massachusetts, bivalve aspergillosis and outbreaks of a trematode parasite were linked to mortality and population declines in blue mussels (Roxanna Smolowitz, New England Aquarium, Boston, MA, personal communication; Scott Haskell, Yuba College, Marysville, $\mathrm{CA}$, personal communication).

In February and March 2006, a large eider mortality event occurred off the southern coast of Cape Cod, MA, with over 300 dead and dying birds on local beaches. An event of similar size, also involving common eiders, occurred off the northern coast of Cape Cod during the fall. The ultimate cause of death remains unknown but is likely multifactorial, with emaciation, toxins in fat stores re-entering circulation as a result of starvation, peritonitis, and immune compromise allowing problems, that would be minor in the absence of parasites, to have devastating effects.

\section{PRESENTATIONS AND PATHOLOGIC FINDINGS}

Based on observation and literature review, there are three major presentations addressed with respect to the common eider:

1. infestation-related emaciation,

2. infestation-related peritonitis, and

3. asphyxiation.

Presumably, other waterbirds with acanthocephaliasis would present similarly.

Infestation-related Emaciation. Wild birds in this condition are found near the beach or in shallow water unable to fly. The birds are approachable and sometimes too weak to exhibit an escape response. Either through direct absorption of amino acids by acanthocephalans, decreased absorption of nutrients by the bird's damaged intestinal mucosa, and/or reduced appetite, emaciation and associated muscle

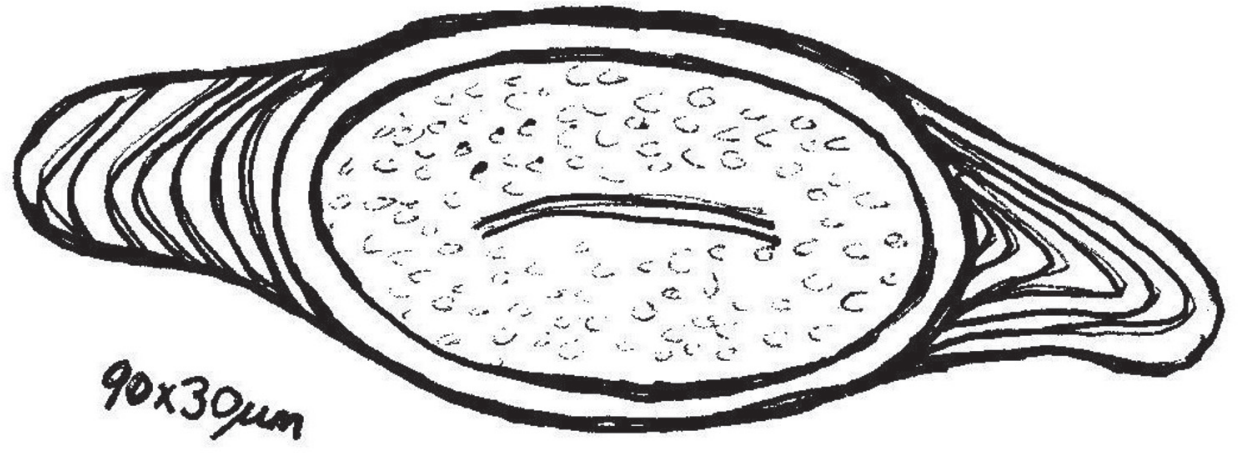

Figure 1. Acanthocephalan egg, occasionally seen on fecal exam. 
atrophy and extreme weakness is common in acanthocephalan infection (Hollmén et al 1999). The eiders present debilitated, emaciated, lethargic, and dehydrated with sunken eyes and tacky mucous membranes. Fecal exams may or may not demonstrate acanthocephalan eggs (Figure 1). As acanthocephalans are predominantly localized in the small intestine, melena (black, tarry feces containing digested blood) may accompany diarrhea. Bile green-stained feces have been noted in rehabilitating birds. Radiography and/or endoscopy may yield findings of marine invertebrates or nothing in the gizzard. Large numbers of snails (commonly periwinkles [Littorina, spp.]) may be found in the gizzard; these are thought to be easy prey items to catch when the bird is weak. If radiograph or necropsy show the ventriculus is full of snails, it strongly indicates the bird is too weak to forage normally. Due to the birds' decreased activity levels and extended time on the beach, external parasites also may be abundant. Birds with acanthocephaliasis, but no clinical signs of disease aside from weight loss, may show abnormalities in chemical profile, including hypoglycemia, an elevated creatine kinase (an enzyme associated with tissue damage), and hypoalbuminemia, without significant changes in PCV (packed cell volume) or total solids. Some birds present with deficiencies in complement components, hemopexin, transferrin, ferritin, plasminogen, fibrinogen, lipoproteins, and immunoglobulins (Hollmén et al 1999). Other biochemical and electrolyte values may be altered as a direct result of associated dehydration. Other clinical findings seen in these birds include hypothermia, anemia, hypercalcemia, hypernatremia, hyperchloremia, an increased percentage of monocytes in white blood cell count, and a generally reactive population of lymphocytes.

On necropsy and pathological examination, the head of the adult $P$. botulus may be seen completely embedded into the intestinal wall, leaving only the body visible, and difficult to detach. The worms are visible, orange and/or white, crescent shaped, and up to $20 \mathrm{~mm}$ (Van Cleave 1916) in length (Figure 2). Proboscis hooks of the adult worm are $16 \mu \mathrm{m}$ long by 7 to $8 \mu \mathrm{m}$ wide (Van Cleave 1916). During the Cape Cod eider die-off of February 2006, necropsy findings from a number of specimens included obstruction/ impaction of small intestine due to high parasite load, emaciation and low body weight, atrophy of skeletal musculature, and occasional peritonitis with serosanguinous (blood-tinged, watery) fluid in the body cavity. Secondary findings included gall bladder distention (consistent with anorexia), atrophy of fat surrounding the heart, liver that is small and dark-tan with sharp edges, and congestion of lungs with blood and fluid, ranging from minimal to marked. Pale kidneys and mucous membranes were commonly found, consistent with anemia caused by the parasites. Histopathology shows that the areas of mucosa surrounding parasite attachment sites are infiltrated with heterophils, mononuclear lymphocytes, and rings of macrophages, all evidence of an inflammatory immune system response (Hollmén et al 1999). Inflammation tends to be limited to tissue immediately adjacent to the parasite's head in mucosa, submucosa, or muscularis layers of the intestinal wall. P. botulus infections reported in a European die-off in 2000 were associated with a multifocal gastrointestinal infectious process varying from pus-producing to localized granulomas to formation of very firm fibrotic tissue. Small spherical bodies resembling bacteria were abundant in lesions while underlying submucosa contained a variety of inflammatory cells (either granulocytes or epithelioid macrophages and multinucleated giant cells) (Kuiken 2001).

Tissues collected from a sample of eight eiders in the 2006 die-off showed mineralization in pulmonary interstitium (structural lung tissue) and renal tubules. 
Small areas of bony cell growth were found in the lungs. Oxalate crystals and evidence of iron accumulation (a result of red blood cell breakdown) were found in the liver. It is speculated these findings are associated with calcium-phosphorous imbalances and chronic parasitism. Acid/base abnormalities resulting from damage to the intestine may contribute to protein binding of calcium that manifests as a change in ionized calcium levels seen on the chemistry panel. Chronic renal interstitial nephritis and tubular necrosis also were seen on histopathology examination.

\section{Infestation-related Peritonitis (with or} without emaciation). Eiders with peritonitis may present similarly to those without peritonitis; however, they also may have life threatening, body-wide infections. These birds exhibit abdominal pain and swelling with ascites, a high white blood cell count, difficulty breathing, and anorexia (Caruso et al 2002). In addition to the pathology listed above, these birds often have adhesions throughout intestines where different segments of intestine have adhered to each other or other structures in the abdominal cavity. Fibrin strands, necrotic or hemorrhagic intestines, congested lungs, and serosanguinous fluid within air sacs has been found on necropsy in these birds.

Asphyxiation. Studies on Steller's eiders (Polysticta stelleri) demonstrate that juveniles in poor body condition consume prey items infected with acanthocephalans, while adults and juveniles in good condition avoid this prey (Bustnes and Galaktionov 2004; Galaktionov 2004). The behavior of consuming infected prey initiates a downward spiral for any bird in poor body condition, leading to severe emaciation. Birds that avoid prey that are common intermediate hosts for acanthocephalans are prone to another clinical presentation from feeding on limited populations of bivalves. Several eiders have presented after choking on food items too large for consumption. The maximum size of bivalves that can be safely consumed by eiders is $7 \mathrm{~cm}$ ( 3 in) in length or $5 \mathrm{~cm}$ ( 2 in) for clams (Nehls 1995). During the Cape Cod die-off, bivalves that were larger than this maximum were found lodged in the mouth/esophagus of the birds and could not be ingested nor ejected from the oral cavity. Because of this, oral cavity examination and palpation of the entire neck is an important part of physical examination, accompanied by radiography to identify any potentially obstructive objects. Birds with this presentation are generally found in acceptable body condition with fewer acanthocephalans than conspecifics. Necropsy of one eider specimen from the
Cape Cod die-off revealed the lungs showed marked pulmonary edema, consistent with asphyxiation. This eider's condition demonstrated fibrotic changes in the liver and chronic infection of ureters and kidneys. Chronic parasitism was thought to be the cause and is a finding relating to this individual bird rather than behavioral reactions to food scarcity.

\section{TREATMENT OPTIONS}

Proper Nutrition. From data gathered in lab experiments and field studies on food deprivation, Wobeser (1981) concluded that waterbirds generally lose about 40 percent of body weight before dying. Kuiken (2001) noted infested eiders in the Wadden Sea (Netherlands) could gain weight and recover in rehabilitation if provided digestible food. Those that died were significantly underweight, ranging from 7 to 52 percent below normal body weight. This should be taken into consideration when attempting to calculate appropriate nutrition for convalescing ducks. Due to the risk of refeeding syndrome following starvation, enteral diets or solid food should be introduced very slowly. Supplementation with vitamin D and high-calcium foods should be avoided to prevent exacerbation of the hypercalcemia.

Antihelminthic Therapy. Currently there are no suggested treatment options for avian acanthocephalan infestation proven effective, but several controversial possibilities for off-label drug use are available:

Dithiazine-experimentally at $25 \mathrm{mg} / \mathrm{kg}$ via crop intubation was found to decrease incidence of worm infection in starlings (Tangredi 1985).

Praziquantel-at $15 \mathrm{mg} / \mathrm{kg} \mathrm{IM}$ was found not to be effective in starlings (Tangredi 1985).

Fenbendazole-PO to common eiders was not conclusive in removing parasites, but had no detrimental effects (Hanssen et al 2003).

Niclosamide-via gavage is recommended, without established efficacy, in some avian species, but may be toxic to some waterfowl (Sanford 1978).

Thiabendazole-PO may partially clear an acanthocephalan infection, reducing worm burden, but potentially toxic to diving ducks (Ballweber 2004).

The benefit hatchling birds may derive from any antihelmintic therapy is questionable. In studies on Eurasian oystercatchers (Haematopus ostralegus), treatment with levamisole, oxyelozanide, and ivermectin was found to decrease hatchling survival (Van Oers et al 2000), though this is potentially a result of interference with development of the immune system rather than direct parasitism effects. 
The following treatments for acanthocephanlan infestation have been successful in non-avian species:

Levamisole-reported successful for treatment in snakes (Kiel and Schmidt 1984).

Albendazole-successful in tamarin monkeys (Weber and Junge 2000).

Loperamide-efficacious in pigs and fish (Mehlhorn et al 1990; Taraschewski et al 1990).

Male fern (Dryopteris filix-mas) extract-more efficacious against primate acanthocephalans when compared to starvation, dithiazine, piperazine, atabrine, or thiabendazole (Moore 1970).

Niclosamide-found a successful treatment in humans (Ikeh et al 1992).

Crude oil-successfully reduced acanthocephalans in Atlantic cod (Gadus morhua) (Khan and Kiceniuk 1983).

Fluid Therapy. Most infected birds present with emaciation and dehydration and appropriate, rapid fluid replacement is one of the most important aspects of treatment. Due to the granulomatous inflammation-induced hypercalcemia, a low calcium fluid such as isotonic saline is preferable to Lactated Ringers ${ }^{\circledR}$ solution (Abbott Laboratories, Abbott Park, IL).

Corticosteroids. Because hypercalcemia is a result of excessive production of 1,25-(OH2)D3 (calcitriol) by activated inflammatory cells commonly seen at the site of acanthocephalan attachment (Sharma 2000), corticosteroids may help reverse this metabolic defect and possibly halt mineralization of tissues and abnormal bony cell growth in lungs. One should note that long-term use of corticosteroids in avian patients is currently heavily debated. While beneficial for antiinflammatory, anticancerous, and immunosuppressive properties, corticosteroids have been documented to cause steroid hepatopathy, gastric ulceration, diabetes- mellitus-like syndromes, lipemia, and intestinal bleeding (Chitty 2001). A steroid-induced immunosuppression may prevent the body from responding appropriately to other pathogens: for this reason, antifungals and antibacterials should be administrated concurrently any time steroids are used. Another caution for corticosteroid use in humans, not yet documented in birds, is the drug's ability to increase the risk of peritonitis (Menegaux et al 1998). Possible dangerous side effects of corticosteroid use must be taken into consideration when evaluating this drug as part of a treatment protocol.
Antibiotics. If peritonitis is present or suspected, suggested treatment includes long-term parenteral (by injection, such as intravenous) antibiotics based on culture and sensitivity. Supportive care is essential, and removal of excess fluid in the abdomen with needle and syringe may be attempted if abdominal pressure increases substantially. This may reduce any associated respiratory difficulties. If toxicity resulting from widespread bacterial infection (endotoxemia) is a probable secondary result of the peritonitis, a onetime injection of dexamethasone may prove useful to mediate some of the inflammatory effects caused by the infection. If this treatment is chosen, an accompanying antifungal should be administered to reduce possibility of Aspergillus spp. overgrowth.

Asphyxiation Cases. Removal of obstruction and subsequent endoscopy to check for other large ingested matter may be the only treatment required. However, the likelihood of receiving a live bird actively choking on a food item is low. Historically, most of these animals have been found washed up on shore rather than brought into a clinic alive. If the bird still has respiratory distress after removal of the obstruction, pulmonary edema may be treated with oxygen and corticosteroids (Orcutt and Flinchum 2001). As discussed previously, steroid use may predispose birds to aspergillosis, which causes additional morbidity in waterfowl, so an accompanying antifungal drug is recommended.

\section{SUMMARY}

Acanthocephalan worms are a common parasite of eiders and other sea ducks, and while they generally do not cause significant morbidity or mortality, at least one species has been associated with several mass mortality events. The most common presentation associated with P. botulus infection is emaciation and debilitation that contributes to a multifactorial cause of death. The apparent intermediate host of the parasite in these die-off events is the shore crab, a prey item that eiders eat in times of blue mussel scarcity. Treatments for acanthocephalan infection are limited and controversial, including various antihelminthics and corticosteroids, along with supportive care and nutrition.

\section{LITERATURE CITED}

Ballweber, L. 2004. Waterfowl parasites. Seminars in Avian and Exotic Pet Medicine. 13(4):197-205.

Bustnes, J., and K. Galaktionov. 2004. Evidence of a state-dependent trade-off between energy intake and parasite avoidance in Steller's eiders. Canadian Journal of Zoology. 82(10):1566-1571. 
Camphuysen, C. J., C. M. Berrevoets, H. J. W. M. Cremers, A. Dekinga, R. Dekker, B. J. Ens, T. M. van der Have, R. K. H. Kats, T. Kuiken, M. F. Leopold, J. Van der Meer, and T. Piersma. 2002. Mass mortality of common eiders (Somateria mollisima) in the Dutch Wadden Sea, winter 1999/2000: Starvation in a commercially exploited wetland of international importance. Biological Conservation. 106(3):303-317.

Caruso, K., R. Cowell, J. Meinkoth, and J. Klaassen. 2002. What is your diagnosis? Abdominal effusion in a bird [Diagnosis: egg yolk peritonitis]. Veterinary Clinical Pathology. 31(3):127-128.

Chitty, J. 2001. Use of corticosteroids in birds. Veterinary Records. 149(16):499-500.

Hanssen, S. A., I. Folstad, K. E. Erikstad, and A. Oksanen. 2003. Costs of parasites in common eiders: effects of antiparasite treatment. Oikos. 100:105-111.

Hollmén, T., J. Lehtonen, S. Sankari, T. Soveri, and M. Hario. 1999. An experimental study on the effects of polymorphiasis in common eider ducklings. Journal of Wildlife Diseases. 35:466-473.

Ikeh, E. I., E. Anosike, and E. Okon. 1992. Acanthocephalan infection in man in northern Nigeria. Journal of Helminthology. 66:241-242.

Khan, R., and J. Kiceniuk. 1983. Effects of crude oils on the gastrointestinal parasites of two species of marine fish. Journal of Wildlife Diseases. 19(3):2538.

Kiel, J., and G. Schmidt. 1984. Mature acanthocephalan infection of king cobras. Avian/Exotic Practice. $1(2): 26-30$.

Kuiken, T. 2001. Pathology of common eiders in the Dutch Wadden Sea in December 1999. Wadden Sea Newsletter. 1:16-18.

Mehlhorn, H., H. Taraschewski, B. Zhao, W. Raether, and T. T. Duggan. 1990. Loperamid, an efficacious drug against the acanthocephalan Macracanthorhynchus hirudinaceus in pigs. Parasitology Research. 76(7):624-6.

Menegaux, F., X. Cherard, B. Wechsler, Z. Boutin, and J. Chigot. 1998. Diffuse peritonitis in steroid-treated patients. Digestive Surgery. 15:247-251.

Moore, J. G. 1970. Epizootic of acanthocephaliasis among primates. Journal of the American Veterinary Medical Association. 157:699-705.

Nehls, G. 1995. Strategien der ernährung und ihre bedeutung für energiehaushalt und Ökologie der Eiderente (Somataria mollissima). PhD thesis, Christian-Albrechts Universität, Kiel.
Orcutt, C., and G. Flinchum. 2001. Corticosteroids in birds. Exotic DVM. 3(5):42-43.

Sanford, S. E. 1978. Mortality in Mute Swans in southern Ontario associated with infestation with the thorny-headed worm, Polymorphus boschadis. Canadian Veterinary Journal. 19:234-236.

Sharma, O. P. 2000. Hypercalcemia in granulomatous disorders: a clinical review. Current Opinions in Pulmonary Medicine. 6(5):442-447.

Sures, B., M. Franken., and H. Taraschewski. 2000. Element concentrations in the archiacanthocephalan Macracanthorhynchus hirudinaceus compared with those in the porcine definitive host from a slaughterhouse in La Paz, Bolivia. International Journal of Parasitology. 30(10):1071-6.

Sures, B. 2002. Competition for minerals between Acanthocephalus lucii and its definitive host perch (Perca fluviatilis). International Journal of Parasitology. 32(9): 1117-22.

Tangredi, B. 1985. Avian acanthocephaliasis. Wildlife Rehabilitation. 4:147-149.

Taraschewski, H. H. Mehlhorn, and W. Raether. 1990. Loperamid, an efficacious drug against fishpathogenic acanthocephalan. Parasitology Research. 76(7):619-23.

Thompson, A. B. 1985. Profilicollis botulus (Acanthocephala) abundance in the eider duck (Somateria mollissima) on the Ythan estuary, Aberdeenshire. Parasitology. 91(3):563-575.

Van Cleave, H. J. 1916. Filicollis botulus N. Sp., With Notes on the Characteristics of the Genus. Transactions of the American Microscopical Society. 35(1):132-133.

Van Oers, K., D. Heg, and S. L. D. Quenec'hdu. 2002. Anthelminthic treatment negatively affects chick survival in the Eurasian Oystercatcher Haematopus ostralegus. Ibis. 144:509-517.

Weber, M., and R. Junge. 2000. Identification and treatment of Moniliformis clarki (Acanthocephala) in cotton-topped tamarins (Saguinus oedipus). Journal of Zoo and Wildlife Medicine. 31:503-507.

Wobeser, G. A., 1981. Diseases of wild waterfowl. Plenum Press: New York, NY.

\section{ACKNOWLEDGEMENTS}

The authors wish to thank Dr. Rebecca Harris and Dr. Julie Ellis for their support and encouragement, the volunteers of the SEANET program for their dedication, WHOI for their collaboration, and Dr. Charles Blend for his expertise regarding parasites. (N. 